\title{
First European Crustacean Conference
}

$\mathrm{T}$ his Conference was held in Paris, from August 31 to September 4, 1992. It was organized in cooperation between the Museum National d'Histoire Naturelle, the Université Pierre et Marie Curie and the Ecole Normale Supérieure.

This multidisciplinary meeting received 429 participants from 42 countries who presented 175 oral communications and 170 posters. Various fundamental or applied aspects of the research on Crustacea were presented in the form of state-of-the-art lectures and specialized communications grouped according to twelve main topics (aquaculture, ecology, ecotoxicology, endocrinology, fisheries, metabolism, neurobiology, nutrition, phylogeny, reproduction, structures, systematics). The Conference included also seven specialized meetings [Sea Lice Workshop, Vth Colloquium Crustacea Decapoda Mediterranea, Thalassinid Workshop, Biogeography Workshop, Aquariology Workshop, XII ${ }^{\mathfrak{e}}$ Réunion des Carcinologistes de Langue Française, general meeting of the Groupe d'Etude et de Réflexion sur l'Evolution des Crustacés (GEREC)]. Copies of the abstracts book may be purchased from: Dr. Pierre Noël, Muséum National d'Histoire Naturelle, Laboratoire de Zoologie (Arthropodes/Crustacés), 61, rue Buffon, 75005 Paris, France).

According to the topics, the abstracts of the communications will appear in several specific Journals, including: Aquatic Living Resources (plenary lectures and a few manuscripts), Crustaceana (systematics, phylogeny, ecology), Cahiers de Biologie Marine (biology, ecology of marine species), Invertebrate Reproduction and Development (reproduction, physiology), Scientia Marina (proceedings of the Colloquium Crustacea Decapoda Mediterranea) and Ellis Horwood Ltd (proceedings of the Sea Lice Workshop).

The organizers wish to thank the following organizations for their generous support : the Université Pierre et Marie Curie (UPMC), the Muséum National d'Histoire Naturelle (MNHN), the Ecole Normale Supérieure (ENS), the Centre National de la Recherche Scientifique (CNRS), the Institut Français de Recherche pour l'Exploitation de la Mer (IFREMER), the Ministère des Affaires Etrangères, the Ministère de la Recherche et de l'Espace, the Ministère de l'Education Nationale et de la Culture, the Ministère de la Francophonie, the Ministère de l'Environnement, the Commission of the European Communities (DG XII and DG XIV, AIR Program), the United Nations Educational Scientific and Cultural Organization (UNESCO), the Crustacean Society, the International Association of Astacology, and the Aquariums Coutant.

A Second European Crustacean Conference is planned, to be held in Belgium in 1995 (or 1996). Those interested can contact Dr. André Péqueux, Laboratoire de Physiologie Animale, Institut Van Beneden, Université de Liège, Quai Van Beneden 22, B-4020 Liège, Belgium. 